S. Endo and T. Miyata

Nagoya Math. J.

Vol. 85 (1982), 231-240

\title{
INTEGRAL REPRESENTATIONS WITH TRIVIAL FIRST COHOMOLOGY GROUPS
}

\author{
SHIZUO ENDO AND TAKEHIKO MIYATA
}

Let $\Pi$ be a finite group and denote by $M_{\Pi}$ the class of finitely generated $Z$-free $Z \Pi$-modules. In [2] we defined a certain equivalence relation on $\boldsymbol{M}_{\Pi}$ and constructed the abelian semigroup $T(I I)$, which was studied in [3] (see [1] and [5], too). In this paper we will define a certain subsemigroup $\tilde{T}(\Pi)$ of $T(\Pi)$ and using this will give a complete answer to a problem raised by $\mathrm{H}$. W. Lenstra, Jr. (for the precise statement see Theorem 2.1 in Section 2).

The contents of this paper were obtained in 1974 and briefly anounced in [4].

\section{$\S 1$.}

Let $I$ be a finite group and $M_{\Pi}$ the class of all $Z \Pi$-lattices, namely, finitely generated $Z$-free $Z \Pi I$-modules. We further define:

$$
\begin{aligned}
& \boldsymbol{H}_{\Pi I}^{i}=\left\{M \in \boldsymbol{M}_{\Pi} \mid H^{i}\left(\Pi^{\prime}, M\right)=0 \text { for every subgroup } \Pi^{\prime} \text { of } \Pi\right\} \\
& \tilde{\boldsymbol{H}}_{I I}=\boldsymbol{H}_{\Pi}^{1} \cap \boldsymbol{H}_{\Pi}^{-1} \\
& \boldsymbol{S}_{I}=\{\text { permutation } Z \Pi \text {-modules }\} \\
& \boldsymbol{D}_{\Pi}=\{\text { direct summands of permutation } Z \Pi \Pi \text {-modules }\} .
\end{aligned}
$$

Define $M^{*}=\operatorname{Hom}_{Z}(M, Z)$. If $M \in \boldsymbol{H}_{\Pi}^{1}$, then $M^{*} \in \boldsymbol{H}_{\Pi}^{-1}$.

Lemma 1.1. For every $M \in M_{I I}$ there exist two exact sequences

(1) $0 \longrightarrow N \longrightarrow S \longrightarrow M \longrightarrow 0, \quad N \in H_{I I}^{1}, S \in S_{I I}$

(2) $0 \longrightarrow M \longrightarrow L \longrightarrow T \longrightarrow 0, \quad L \in H_{\Pi}^{1}, T \in S_{\Pi}$.

Proof. (1) is Lemma 1.1 in [3].

(2) $M$ can be imbedded in a suitable free $Z \Pi$-module $F$ such that $F / M$ is $Z$-free. By (1) there is an exact sequence $0 \rightarrow N^{\prime} \rightarrow T \rightarrow F / M \rightarrow 0$ with 
$N^{\prime} \in \boldsymbol{H}_{I I}^{1}$ and $T \in \boldsymbol{S}_{\Pi}$. Taking the pullback of $F \rightarrow F / M$ we have an exact $\uparrow$

sequence $0 \rightarrow M \rightarrow F \oplus N^{\prime} \rightarrow T \rightarrow 0$. This completes the proof.

Lemma 1.2. Let $\Pi_{0}$ be a subgroup of $\Pi$. Consider an exact sequence

$$
0 \longrightarrow M \longrightarrow N \stackrel{\phi}{\longrightarrow} Z \Pi / \Pi_{0} \oplus L \longrightarrow 0, \quad M, N, L \in M_{\Pi} .
$$

If $H^{1}\left(\Pi_{0}, M\right)=0$, then $N$ has a direct sum decomposition $N=N_{1} \oplus N_{2}$ such that the restriction $\phi$ to $N_{1}$ is an isomorphism onto $Z \Pi / \Pi_{0}$.

Especially $\operatorname{Ext}_{Z I I}^{1}(L, M)=0$ for $L \in D_{\Pi I}$ and $M \in \boldsymbol{H}_{\Pi}^{1}$.

Proof. Let $n$ be a fixed element of $\phi^{-1}\left(\Pi_{0} / \Pi_{0}\right)$. Then $\sigma n-n \in M$ for every $\sigma \in \Pi_{0}$, i.e., $\sigma n-n, \sigma \in \Pi_{0}$ is a cocycle of $\Pi_{0}$ with values in $M$. By the assumption there is an $m \in M$ such that $\sigma n-n=\sigma m-m$ for all $\sigma \in$ $\Pi_{0}$. Set $N_{1}=Z \Pi \cdot(n-m)$ and $N_{2}=\phi^{-1}(L)$. Clearly $N=N_{1} \oplus N_{2}$ and $\phi$ : $N_{1} \rightarrow Z \Pi / \Pi_{0}$ is an isomorphism.

Remark 1.3. Let $p$ be a prime. $Z_{p}$ denotes the completion of $Z$ at $p$. For a $p$-group $\Pi$, a more precise statement than Lemma 1.2 holds. Namely, consider an exact sequence: $0 \rightarrow M \rightarrow S \stackrel{\phi}{\longrightarrow} Z_{p} \Pi / \Pi_{0} \oplus T \rightarrow 0$ with $M, a Z_{p} \Pi$-lattice and $S, T$, permutation $Z_{p} \Pi$-modules. If $H^{1}\left(\Pi_{0}, M\right)=0$, then $S$ has a direct sum decomposition $S=S_{1} \oplus S_{2}$ such that $S_{1}$ and $S_{2}$ are permutation $Z_{p} \Pi$-modules and $\phi: S_{1} \rightarrow Z_{p} \Pi / \Pi_{0}$ is an isomorphism.

The proof follows from the similar argument as above, Krull-Schmidt's theorem and the fact that $Z_{p} \Pi / \Pi^{\prime}$ is an indecomposable $Z_{p} \Pi$-module for an arbitrary subgroup $\Pi^{\prime}$ of $\Pi$.

For $M, N \in M_{I I}$ we define $M \equiv N$ by the existence of two exact sequences:

$$
\begin{aligned}
& 0 \longrightarrow M \longrightarrow X \longrightarrow S \longrightarrow 0 \\
& 0 \longrightarrow N \longrightarrow X \longrightarrow T \longrightarrow 0
\end{aligned}
$$

with $X \in M_{I I}$ and $S, T \in \boldsymbol{S}_{\Pi}$. Lemmas 1.1 and 1.2 show that " $\equiv "$ is an equivalence relation on $\boldsymbol{M}_{\Pi}$. Now we define

$$
T(\Pi)=M_{\Pi} /(\equiv)
$$

where the addition is introduced to $T(\Pi)$ by the direct sum. It is easy to check that this semigroup coincides with our old $T(\Pi)$ defined in [2]. By Lemma $1.1 T(\Pi)$ is generated by $\boldsymbol{H}_{\Pi}^{1} . \quad \tilde{T}(\Pi)$ is defined to be the subsemigroup of $T(I)$ which is generated by $\boldsymbol{H}_{I I}^{-1}$ (note that this is already 
generated by $\left.\tilde{H}_{I}\right)$. We denote by $T^{g}(I)$ the set of invertible elements in $T(\Pi)$. This is clearly an abelian group and is known to be generated by $\boldsymbol{D}_{\Pi}$. Hence $T^{g}(\Pi)$ is finitely generated by [3], (1.5).

$\S 2$.

The aim of this paper is to prove the following

Theorem 2.1. Let $\Pi$ be a finite group and let $\Pi^{p}$ be a Sylow p-subgroup of $\Pi$ for each prime $p$. Then the following statements are equivalent:

(1) $\tilde{H}_{I I}=D_{I I}$, i.e., $\tilde{T}(I)$ is a group,

(2) $\Pi^{p}$ is cyclic for each odd prime $p$, and $\Pi^{2}$ is cyclic or dihedral (including Klein's four group).

This gives a complete answer to a problem which was raised by $H$. W. Lenstra, Jr.

In this section we will give an outline of the proof of the theorem and postpone proofs of technical lemmas to later sections.

LeMma 2.2. (1) If $\tilde{\boldsymbol{H}}_{I I}=D_{I I}$, then $\tilde{\boldsymbol{H}}_{I^{\prime}}=D_{I^{\prime}}$ for any subgroup $\Pi^{\prime}$ of $\Pi$.

(2) $\tilde{H}_{I I}=D_{I I}$ if and only if $\tilde{\boldsymbol{H}}_{I^{p}}=\boldsymbol{D}_{I^{p}}$ for every prime $p$.

Proof. (1) If $M \in \tilde{\boldsymbol{H}}_{\Pi^{\prime}}$, then $Z \Pi \otimes_{Z \Pi^{\prime}} M \in \tilde{\boldsymbol{H}}_{\Pi I}=\boldsymbol{D}_{\Pi !}$. Since $M$ is a direct summand of $Z \Pi \otimes_{Z \Pi^{\prime}} M$ as a $Z \Pi^{\prime}$-module, we see that $M \in D_{I I^{\prime}}$.

(2) If $M \in \tilde{\boldsymbol{H}}_{I}$, then for any prime $p, M \in \tilde{\boldsymbol{H}}_{I^{p}}$. If $\tilde{\boldsymbol{H}}_{\Pi^{p}}=D_{\Pi^{p}}$ for every prime $p$, then clearly $M \in D_{I I}$ by [3], (1.4). The converse follows from (1).

By this lemma it suffices to prove the theorem for a $p$-group $\Pi$.

Let $\Pi$ be a finite group and let $I_{I I}$ be the augmentation ideal of $Z \Pi$, i.e., $I_{\Pi}=\operatorname{Ker} \varepsilon_{\Pi}$, where $\varepsilon_{\Pi}: Z \Pi \rightarrow Z$ denotes the augmentation map. We have an exact sequence

$$
0 \longrightarrow I_{I I} \otimes_{Z} I_{I I} \longrightarrow Z \Pi^{(n-1)} \longrightarrow I_{I} \longrightarrow 0, \quad n=|\Pi| .
$$

We define $L_{I I}=\left(I_{I} \otimes_{Z} I_{I I}\right)^{*}=\operatorname{Hom}_{Z}\left(I_{I} \otimes_{Z} I_{I I}, Z\right)$, then $\left[L_{I I}\right] \in \tilde{T}(\Pi)$. It is routine to show

LEMma 2.3. $L_{\Pi} \in \boldsymbol{H}_{\Pi}^{-1} \cap \boldsymbol{H}_{I I}^{-3}, H^{-2}\left(\Pi, L_{\Pi}\right) \cong Z /|\Pi| \boldsymbol{Z}, \hat{H}^{0}\left(\Pi, L_{\Pi}\right) \cong \Pi /[\Pi, \Pi]$ $([\Pi, \Pi]$ denotes the commutator subgroup of $\Pi)$ and $H^{1}\left(\Pi, L_{\Pi}\right) \cong H^{2}(\Pi, Q / Z)$ (the Schur multiplier of $\Pi$ ).

This lemma will be used in Section 3.

LEMMA 2.4. Let $\Pi$ be one of the following groups: 
(1) $Z / 2 Z \times Z / 2 Z \times Z / 2 Z$,

(2) the quarternion group $H_{2}$ of order 8 ,

(3) $Z / p Z \times Z / p Z$, where $p$ is an odd prime,

(4) $Z / 4 Z \times Z / 2 Z$.

Then $\left[L_{\Pi}\right] \oplus T^{g}(\Pi)$. Especially, $\tilde{T}(\Pi)$ is not a group.

This lemma will be proved in Section 3. It is easy to show the following

Lemma 2.5. Let $\Pi$ be a finite 2-group of order $\geqq 8$. Assume that there exists no subgroup of $\Pi$ isomorphic to one of the following:

(1) $Z / 2 Z \times Z / 2 Z \times Z / 2 Z$,

(2) the quarternion group $H_{2}$ of order 8 ,

(3) $Z / 4 Z \times Z / 2 Z$,

then II is cyclic or dihedral.

For an odd prime $p$, if a $p$-group $\Pi$ has no subgroup isomorphic to $\boldsymbol{Z} \mid p \boldsymbol{Z} \times \boldsymbol{Z} / p \boldsymbol{Z}$, then $\Pi$ is cyclic. Hence the implication (1) $\Rightarrow(2)$ in Theorem 2.1 follows from Lemmas 2.2, 2.4 and 2.5.

We denote by $D_{2 \ell}, \ell=1$, the dihedral group of order $2^{\ell+1}$, i.e.,

$$
D_{2^{\ell}}=\left\langle\sigma, \tau \mid \sigma^{2^{\ell}}=\tau^{2}=1, \tau^{-1} \sigma \tau=\sigma^{-1}\right\rangle
$$

$D_{2}$ is Klein's four group $Z / 2 Z \times Z / 2 Z$. From now on we assume $\ell \geqq 2$. The key point in proving the implication $(2) \Rightarrow(1)$ in Theorem 2.1 is that a $Z D_{2 c}$-lattice with no non-zero element fixed by $\left\langle\sigma^{2^{\ell-1}}\right\rangle$, the center of $D_{2^{\ell}}$, can be completely classified locally. To state the classification explicitly we will prepare a few more notations.

Denote by $\zeta$ a primitive $2^{\ell}$-th root of unity and put $R_{\ell}=Z[\zeta]$ and $\mathscr{P}_{\ell}$ $=(\zeta-1) R_{\ell}$. Define the action of $\langle\tau\rangle$ on $R_{\ell}$ by $\tau(\zeta)=\zeta^{-1}$, i.e., identify $\tau$ with the complex conjugation. Then $\mathscr{P}_{\ell}$ is the unique ambiguous prime ideal of $R_{\ell}$ ramified over $Z$. We define $\Lambda_{\ell}=Z D_{2^{\ell}} /\left(\sigma^{2^{\ell-1}}+1\right)$. Then $\Lambda_{\ell}$ is isomorphic to the trivial crossed product of $R_{\ell}$ and $\langle\tau\rangle . \quad R_{\ell}$ and $\mathscr{P}_{\ell}$ clearly can be regarded as $\Lambda_{\ell}$-modules naturally. $\Lambda_{\ell}, R_{\ell}$ and $\mathscr{P}_{\ell}$ are quasipermutation modules ( $M \in M_{I I}$ is a quasi-permutation module if there exists permutation $Z \Pi$-modules $S_{1}, S_{2}$ such that $0 \rightarrow M \rightarrow S_{1} \rightarrow S_{2} \rightarrow 0$ is exact).

Lemma 2.6. Let $M$ be a finitely generated $Z$-free $\Lambda_{\ell}$-module. Then $M$ has the same genus as $\Lambda_{\ell}^{(r)} \oplus R_{\ell}^{(s)} \oplus \mathscr{P}_{\ell}^{(t)}$ for some $r, s, t \geqq 0$, i.e., these two modules are locally isomorphic and hence $[M] \in T^{g}\left(D_{2^{t}}\right)$. 
Using the lemma we can prove

Lemma 2.7. $\tilde{T}\left(D_{2^{\ell}}\right)$ is a group.

In [3] we proved that let $\Pi$ be a $p$-group with $p$ odd prime, then $T(\Pi)$ is a group if and only if $\Pi$ is cyclic. Lemma 2.4 shows that the following statements are equivalent for a $p$-group $\Pi$ with $p$ odd prime:

(i) $\Pi$ is cyclic,

(ii) $T(I I)$ is a group,

(iii) $\tilde{T}(I I)$ is a group.

The implication $(2) \Rightarrow(1)$ in Theorem 2.1 follows from this observation and Lemmas 2.2 and 2.7 .

Remark 2.8. In [3] we proved that $T(\Pi)$ is a group if and only if $\left[I_{I I}^{*}\right] \in T^{g}(\Pi)$. An analogous statement for $\tilde{T}(\Pi)$ can also be proved, i.e., $\tilde{T}(\Pi)$ is a group if and only if $\left[L_{I I}\right]=\left[\left(I_{I} \otimes_{Z} I_{I I}\right)^{*}\right] \in T^{g}(\Pi)$.

\section{$\S 3$.}

Proof of Lemma 2.4. Throughout this section we assume that $I$ is a $p$-group. It suffices to show that if $\left[L_{\Pi}\right] \in T^{g}(\Pi)$, then $\Pi$ is not isomorphic to any group listed in Lemma 2.4. Assume that $\left[L_{\Pi}\right] \in T^{g}(\Pi)$. Since $M$ belongs to $D_{I I}$ if and only if $Z_{p} \otimes_{Z} M$ is a permutation $Z_{p}$-module Lemma 1.1 shows that there is an exact sequence:

$$
0 \longrightarrow Z_{p} \otimes_{Z} L_{I I} \longrightarrow S_{1} \longrightarrow S_{2} \longrightarrow 0
$$

where $S_{1}$ and $S_{2}$ are permutation $Z_{p} \Pi$-modules. Remark 1.3 allows us to assume that $S_{2}$ has no direct summand isomorphic to $Z_{p} \Pi / \Pi_{0}$ for any cyclic subgroup $\Pi_{0}$ of $\Pi$ (note that $L_{\Pi} \in \boldsymbol{H}_{\Pi}^{-1}$ and hence $H^{1}\left(\Pi_{0}, L_{\Pi}\right)=0$ by periodicity). Taking a long cohomology sequence and noting Lemma 2.3, we obtain an exact sequence:

$$
\begin{aligned}
0 \longrightarrow H^{-3}\left(\Pi, S_{1}\right) \longrightarrow & H^{-3}\left(\Pi, S_{2}\right) \longrightarrow Z /|\Pi| Z \\
& \longrightarrow H^{-2}\left(\Pi, S_{1}\right) \longrightarrow H^{-2}\left(\Pi, S_{2}\right) \longrightarrow 0 .
\end{aligned}
$$

(1) Let $\Pi$ be $Z / 2 Z \times Z / 2 Z \times Z / 2 Z$. In this case all cohomology groups appearing in (3.2) are annihilated by 2 . This is a contradiction.

(2) Let $\Pi$ be the quaternion group of order 8 . Then $H^{-2}(I, S)$ is annihilated by 4 and $H^{-3}(\Pi, S) \cong H^{-1}(\Pi, S)=0$ for all permutation $Z_{2} \Pi$ modules $S$. This contradicts (3.2).

(3) Let $I I$ be $\boldsymbol{Z} / p \boldsymbol{Z} \times \boldsymbol{Z} / p \boldsymbol{Z}$, where $p$ is an odd prime. In this case we 
can put $S_{2}=Z_{p}^{(n)}, S_{1}=Z_{p}^{(m)} \oplus T$, where $T$ is a permutation $Z_{p} \Pi$-module having no direct summand isomorphic to $Z_{p}$ since a proper subgroup of $\Pi$ is cyclic. We derive another cohomology sequence from (3.1):

$$
\begin{aligned}
0 \longrightarrow \hat{H}^{0}\left(\Pi, Z_{p} \otimes_{Z} L_{\Pi}\right) & \longrightarrow \hat{H}^{0}\left(\Pi, Z_{p}^{(m)} \oplus T\right) \\
& \longrightarrow \hat{H}^{0}\left(\Pi, Z_{p}^{(n)}\right) \longrightarrow H^{1}\left(\Pi, Z_{p} \otimes_{Z} L_{\Pi}\right) \longrightarrow 0 .
\end{aligned}
$$

From this we obtain an exact sequence:

$$
0 \longrightarrow(\boldsymbol{Z} / p \boldsymbol{Z})^{(2)} \longrightarrow\left(\boldsymbol{Z} / p^{2} \boldsymbol{Z}\right)^{(m)} \oplus \hat{H}^{0}(\Pi, T) \longrightarrow\left(\boldsymbol{Z} / p^{2} \boldsymbol{Z}\right)^{(n)} \longrightarrow \boldsymbol{Z} / p \boldsymbol{Z} \longrightarrow 0 \text {. }
$$

Hence $n-1=m$ or $m=n$ because $\hat{H}^{0}(\Pi, T)$ is annihilated by $p$. Let $\Pi^{\prime} \neq 1$ be a cyclic subgroup of $\Pi$. From (3.1) we get an exact sequence:

$$
0 \longrightarrow Z / p \boldsymbol{Z} \longrightarrow(\boldsymbol{Z} / p \boldsymbol{Z})^{(m)} \oplus \hat{H}^{0}\left(\Pi^{\prime}, T\right) \longrightarrow(\boldsymbol{Z} / p \boldsymbol{Z})^{(n)} \longrightarrow 0 \text {. }
$$

Hence $\hat{H}^{0}\left(\Pi^{\prime}, T\right) \cong(\boldsymbol{Z} / p \boldsymbol{Z})^{(2)}$ if $n-1=m$ or $\cong \boldsymbol{Z} / p \boldsymbol{Z}$ if $n=m$. On the other hand since $T$ has no direct summand isomorphic to $Z_{p}$, we see that $\operatorname{rank}_{Z_{p}} \hat{H}^{0}\left(\Pi^{\prime}, T\right)$ is divisible by $p$. This is a contradiction.

(4) Let $\Pi$ be $Z / 4 Z \times Z / 2 Z=\langle\sigma\rangle \times\langle\tau\rangle$. In this case (3.1) looks like

$$
\begin{aligned}
0 \longrightarrow Z_{p} \otimes_{Z} L_{I I} \longrightarrow Z_{p}^{(\ell)} \oplus & \left(Z_{p} \Pi /\left\langle\sigma^{2}, \tau\right\rangle\right)^{(m)} \oplus T \\
& \longrightarrow Z_{p}^{\left(\ell^{\prime}\right)} \oplus\left(Z_{p} \Pi /\left\langle\sigma^{2}, \tau\right\rangle\right)^{\left(m^{\prime}\right)} \longrightarrow 0
\end{aligned}
$$

where $T=\oplus_{\Pi^{\prime} \leqq I, \Pi^{\prime}: \operatorname{cyclic}}\left(Z_{p} \Pi / \Pi^{\prime}\right)^{\left(n \Pi^{\prime}\right)}$.

Set $\Pi_{0}=\left\langle\sigma^{2}, \tau\right\rangle$. Then it is easy to check that

$$
0 \longrightarrow Z_{p} \otimes_{Z} L_{\Pi_{0}} \longrightarrow Z_{p} \Pi_{0} \mid\left\langle\sigma^{2}\right\rangle \oplus Z_{p} \Pi_{0} /\langle\tau\rangle \oplus Z_{p} \Pi_{0} /\left\langle\sigma^{2} \tau\right\rangle \stackrel{\varepsilon}{\longrightarrow} Z_{p} \longrightarrow 0
$$

is exact, where $\varepsilon$ is the sum of augmentation maps. It is also easy to see that

$$
\left(Z_{p} \otimes_{Z} L_{\Pi}\right) \oplus F_{1} \cong\left(Z_{p} \otimes_{Z} L_{I_{0}}\right) \oplus F_{2}
$$

as $Z_{p} \Pi_{0}$-modules with suitable free $Z_{p} \Pi_{0}$-modules $F_{1}, F_{2}$. Taking the pushout of (3.3) and (3.4), we have

$$
\begin{array}{r}
Z_{p}^{(\ell)} \oplus\left(Z_{p} \Pi \mid\left\langle\sigma^{2}, \tau\right\rangle\right)^{(m)} \oplus T \oplus F_{1} \oplus Z_{p} \\
\cong Z_{p}^{\left(\ell^{\prime}\right)} \oplus\left(Z_{p} \Pi \mid\left\langle\sigma^{2}, \tau\right\rangle\right)^{\left(m^{\prime}\right)} \oplus F_{2} \oplus Z_{p} \Pi_{0} \mid\left\langle\sigma^{2}\right\rangle \\
\oplus Z_{p} \Pi_{0}\left|\langle\tau\rangle \oplus Z_{p} \Pi_{0}\right|\left\langle\sigma^{2} \tau\right\rangle
\end{array}
$$

as $Z_{p} \Pi_{0}$-modules. Simple computations show that as a $Z_{p} \Pi_{0}$-module $T$ has even number of direct summands isomorphic to $Z_{p} \Pi_{0} /\langle\tau\rangle$. This contradicts (3.5). 
$\S 4$.

Proofs of Lemmas 2.6 and 2.7. First we consider proof of Lemma 2.6.

Lemma 4.1. Let $R$ be a ring and let $A_{1}, A_{2}$ and $X$ be $R$-modules with the following properties:

(1) $0 \rightarrow A_{i} \rightarrow X \rightarrow A_{i} \rightarrow 0(i=1,2)$ are non-split exact sequences.

(2) $\operatorname{Ext}_{R}^{1}\left(A_{i}, A_{i}\right) \cong Z / 2 Z, \operatorname{Ext}_{R}^{1}\left(A_{i}, X\right) \cong \operatorname{Ext}_{R}^{1}\left(X, A_{i}\right)=0 i=1,2$ and $\operatorname{Ext}_{R}^{1}\left(A_{1}, A_{2}\right) \cong \operatorname{Ext}_{R}^{1}\left(A_{2}, A_{1}\right) \cong \operatorname{Ext}_{R}^{1}(X, X)=0$.

Consider an extension

$$
0 \longrightarrow A_{1}^{\left(s_{1}\right)} \oplus A_{2}^{\left(s_{2}\right)} \oplus X^{(t)} \longrightarrow Y \longrightarrow A_{1}^{\left(s_{1}^{\prime}\right)} \oplus A_{2}^{\left(s_{2}^{\prime}\right)} \oplus X^{\left(t^{\prime}\right)} \longrightarrow 0
$$

then $Y$ is of the same type, i.e., $Y=A_{1}^{\left(s_{1}^{\prime \prime}\right)} \oplus A_{2}^{\left(s_{2}^{\prime \prime}\right)} \oplus X^{\left(t^{\prime \prime}\right)}$.

Proof is easy.

We have the exact sequences:

$$
\begin{aligned}
& 0 \longrightarrow \Lambda_{\ell}(\tau-1) \longrightarrow \Lambda_{\ell} \longrightarrow \Lambda_{\ell}(\tau+1) \longrightarrow 0 \\
& 0 \longrightarrow \Lambda_{\ell}\left(\tau-\zeta^{-1}\right) \longrightarrow \Lambda_{\ell} \longrightarrow \Lambda_{\ell}(\tau+\zeta) \longrightarrow 0 .
\end{aligned}
$$

Define the homomorphism $\phi: R_{\ell} \rightarrow \Lambda_{\ell}(\tau+1)$ (resp. $\phi^{\prime}: \mathscr{P}_{\ell} \rightarrow \Lambda_{\ell}(\tau+\zeta)$ ) by $\phi(x)=x(\tau+1)\left(\right.$ resp. $\left.\phi^{\prime}(x(\zeta+1))=x(\tau+\zeta)\right)$ for any $x \in R_{\ell}$. Then $\phi$ and $\phi^{\prime}$ are $\Lambda_{\ell}$-homomorphisms. Put $\omega=(1+\zeta)(1-\zeta)^{-1}$ and $\omega^{\prime}=(1+\zeta)\left(1-\zeta^{-1}\right)^{-1}$ $\in U\left(R_{\ell}\right)$ and define the homomorphism $\psi: R_{\ell} \rightarrow \Lambda_{\ell}(\tau-1)$ (resp. $\psi^{\prime}: \mathscr{P}_{\ell} \rightarrow$ $\left.\Lambda_{\ell}\left(\tau-\zeta^{-1}\right)\right)$ by $\psi(x)=x \omega(\tau-1)$ (resp. $\left.\psi^{\prime}(x(\zeta+1))=x \omega^{\prime}\left(\tau-\zeta^{-1}\right)\right)$ for any $x \in R_{\ell}$. We easily show that both $\psi$ and $\psi^{\prime}$ are $\Lambda_{\ell}$-isomorphisms. Therefore, we have

$$
\Lambda_{\ell}(\tau+1) \cong \Lambda_{\ell}(\tau-1) \cong R_{\ell} \text { and } \Lambda_{\ell}(\tau+\zeta) \cong \Lambda_{\ell}\left(\tau-\zeta^{-1}\right) \cong \mathscr{P}_{\ell}
$$

as $\Lambda_{\ell}$-modules. Thus we get the non-split exact sequences:

$$
\begin{aligned}
& 0 \longrightarrow R_{\ell} \longrightarrow \Lambda_{\ell} \stackrel{f}{\longrightarrow} R_{\ell} \longrightarrow 0 \\
& 0 \longrightarrow \mathscr{P}_{\ell} \longrightarrow \Lambda_{\ell} \stackrel{f^{\prime}}{\longrightarrow} \mathscr{P}_{\ell} \longrightarrow 0
\end{aligned}
$$

where $f$ (resp. $f^{\prime}$ ) is defined by $f(x+y \tau)=x+y$ (resp. $f^{\prime}(x+y \tau)=$ $\left.\left(x+y \zeta^{-1}\right)(\zeta+1)\right)$. From (4.1) we get the exact sequence:

$$
\operatorname{Hom}_{A_{\ell}}\left(R_{\ell}, \Lambda_{\ell}\right) \stackrel{\tilde{f}}{\longrightarrow} \operatorname{Hom}_{A_{\ell}}\left(R_{\ell}, R_{\ell}\right) \longrightarrow \operatorname{Ext}_{\Lambda_{\ell}}^{1}\left(R_{\ell}, R_{\ell}\right) \longrightarrow 0 .
$$

Every $g \in \operatorname{Hom}_{A_{\ell}}\left(R_{\ell}, R_{\ell}\right)$ can be identified with $g(1) \in R_{\ell}$. Then we have

$$
\operatorname{Hom}_{\iota_{\ell}}\left(R_{\ell}, R_{\ell}\right)=\left\{x \in R_{\ell} \mid \bar{x}=x\right\} \text { and } \operatorname{Im} \tilde{f}=\left\{x+\bar{x} \mid x \in R_{\ell}\right\}
$$


where $\bar{x}$ is the complex conjugation of $x \in R_{\ell}$. By a direct computation it is seen that

$$
\operatorname{Ext}_{A_{\ell}}^{1}\left(R_{\ell}, R_{\ell}\right) \cong \operatorname{Hom}_{A_{\ell}}\left(R_{\ell}, R_{\ell}\right) / \operatorname{Im} \tilde{f} \cong Z / 2 Z .
$$

Similarly we can show that

$$
\operatorname{Ext}_{\Lambda_{\ell}}^{1}\left(\mathscr{P}_{\ell}, \mathscr{P}_{\ell}\right) \cong Z / 2 Z \text { and } \operatorname{Ext}_{A_{\ell}}^{1}\left(R_{\ell}, \mathscr{P}_{\ell}\right) \cong \operatorname{Ext}_{\Lambda_{\ell}}^{1}\left(\mathscr{P}_{\ell}, R_{\ell}\right)=0 .
$$

This shows that $R_{\ell}, \mathscr{P}_{\ell}$ and $\Lambda_{\ell}$ satisfy the conditions in Lemma 4.1.

We localize everything at 2 and denote them by the same notations. Let $\Omega_{\ell}$ be a maximal order in $Q_{2} \Lambda_{\ell}$ containing $\Lambda_{\ell}$ and let $M$ be a $\Lambda_{\ell}$-lattice. Then we can write uniquely $\Omega_{\ell} M \cong \Omega_{\ell} R_{\ell}^{(n)}, n \geqq 0$. We call $n$ the rank of $M$. We will prove the assertion by induction on $n$. It is noted that any ambiguous ideal of $R_{\ell}$ is isomorphic to $R_{\ell}$ or $\mathscr{P}_{\ell}$. If $n=1, M$ is isomorphic to an ambiguous ideal of $R_{\ell}$ and so $M \cong R_{\ell}$ or $\mathscr{P}_{\ell}$. Now we assume that $n \geqq 2$ and the assertion is true for $N$ with rank $N \leqq n-1$. We can write $\Omega_{\ell} M=L_{1} \oplus L_{2}$, where $L_{1} \cong \Omega_{\ell} R_{\ell}^{(n-1)}$ and $L_{2} \cong \Omega_{\ell} R_{\ell}$. Put $N=M \cap L_{1}$ and $M^{\prime}=M / N$. Then by the induction hypothesis we have $N \cong \Lambda_{\ell}^{(r)} \oplus$ $R_{\ell}^{(s)} \oplus \mathscr{P}_{\ell}^{(t)}$ for some $r, s$, and $t$. Since $M^{\prime} \cong R_{\ell}$ or $\mathscr{P}_{\ell}$, we have $M \cong \Lambda_{\ell}^{\left(r^{\prime}\right)}$ $\oplus R_{\ell}^{\left(\prime^{\prime}\right)} \oplus \mathscr{P}_{\ell}^{\left(t^{\prime}\right)}$ by Lemma 4.1. This completes the proof.

Finally we shall prove Lemma 2.7. If $I$ is cyclic this assertion was proved in [3]. Therefore we only need to consider the case where $\Pi$ is dihedral, i.e.,

$$
\Pi=D_{2^{\ell}}=\left\langle\sigma, \tau \mid \sigma^{2^{\ell}}=\tau^{2}=1, \tau^{-1} \sigma \tau=\sigma^{-1}\right\rangle, \quad \ell \geqq 1 .
$$

We will prove the assertion by induction on $\ell$.

We first assume that $\ell=1$. In this case $D_{2}$ is Klein's four group. Let $L \in \tilde{\boldsymbol{H}}_{I}$. Define the homomorphism $N_{\sigma}: L \rightarrow L$ by $N_{\sigma}(u)=(1+\sigma) u$ for $u \in L$. Then $H^{-1}(\langle\sigma\rangle, L)=\operatorname{Ker} N_{o} /(\sigma-1) L=0$ and hence we have the exact sequence

$$
0 \longrightarrow(\sigma-1) L \longrightarrow L \longrightarrow \operatorname{Im} N_{\sigma} \longrightarrow 0 \text {. }
$$

Since $\hat{H}^{0}(\Pi,(\sigma-1) L)=0$, we have $H^{-1}\left(\Pi, \operatorname{Im} N_{\sigma}\right)=0$. Since $\operatorname{Im} N_{\sigma}$ can be regarded as a $Z \Pi \mid\langle\sigma\rangle$-module and $Z$-free, we easily see that $H^{-1}(\Pi \mid\langle\sigma\rangle$, $\operatorname{Im} N_{\sigma}$ ) $=0$. This shows that $\operatorname{Im} N_{\sigma}$ is a permutation $Z \Pi /\langle\sigma\rangle$-module. We obtain that

$$
(\sigma-1) L \cong(Z \Pi /(\sigma+1, \tau-1))^{(r)} \oplus(Z \Pi /(\sigma+1, \sigma \tau-1))^{(s)} \oplus(Z \Pi /(\sigma+1))^{(t)}
$$

for some $r, s, t \geqq 0$ and therefore $[(\sigma-1) L]$ is zero in $T(I)$. Hence $[L]=$ 
$[(\sigma-1) L]=0$. This shows $\tilde{T}(I)=0$, or $\tilde{\boldsymbol{H}}_{I I}=\boldsymbol{D}_{\Pi}$. Next assume that $\ell$ $\geqq 2$ and the assertion is true for $D_{2^{j}}, j \leqq \ell-1$. Let $L \in \tilde{\boldsymbol{H}}_{D^{2} \ell}$. Then we have the exact sequence

$$
0 \longrightarrow\left(\sigma^{\imath-1}-1\right) L \longrightarrow L \longrightarrow\left(\sigma^{2^{\ell-1}}+1\right) L \longrightarrow 0 .
$$

Put $L^{\prime}=\left(\sigma^{2^{6-1}}-1\right) L$ and $L^{\prime \prime}=\left(\sigma^{2^{\ell-1}}+1\right) L$. If $\Pi^{\prime}$ is a subgroup of $\Pi=$ $D_{2^{\ell}}$ containing $\left\langle\sigma^{2^{\ell-1}}\right\rangle$, then $\hat{H}^{0}\left(\Pi^{\prime}, L^{\prime}\right)=0$ hence $H^{-1}\left(\Pi^{\prime}, L^{\prime \prime}\right)=0$. Since $L^{\prime \prime}$ can be regarded as $Z$-free $Z \Pi \mid\left\langle\sigma^{2^{\ell-1}}\right\rangle$-module, we have $H^{-1}\left(\Pi^{\prime} \mid\left\langle\sigma^{2^{2-1}}\right\rangle, L^{\prime \prime}\right)$ $=0$. Hence $L^{\prime \prime} \in \boldsymbol{H}_{\Pi}^{-1} \mid\left\langle{\sigma^{2}}^{c-1}\right\rangle$. By Lemma 2.6 there are a permutation $Z \Pi$ module $S$ and a $Z M$-lattice $T$ locally isomorphic to a permutation module such that

$$
0 \longrightarrow L^{\prime} \longrightarrow S \longrightarrow T \longrightarrow 0
$$

is exact. Taking the pushout of $L^{\prime} \rightarrow L$ we get the following commutative diagram with exact rows and columns:

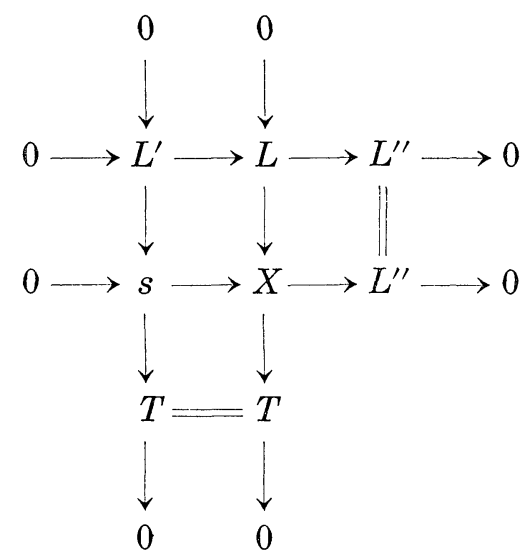

Since $L^{\prime \prime} \in \boldsymbol{H}_{\left.I I / \alpha^{2} e^{-1}\right\rangle}^{-1} \subseteq \boldsymbol{H}_{I I}^{-1}$ and $L \in \boldsymbol{H}_{I I}^{1}$, we have $X \cong L \oplus T \cong S \oplus L^{\prime \prime}$. This shows that $L^{\prime \prime} \in \boldsymbol{H}_{\Pi}^{1}$ and hence $L^{\prime \prime} \in \tilde{\boldsymbol{H}}_{\Pi /\left\langle\mathrm{v}^{2} \mathrm{l}\right.}$. By the induction hypothesis $L^{\prime \prime} \in D_{I /\left\langle\sigma 2^{\ell-1}\right\rangle} \subseteq D_{I}$, thus $L \in D_{\Pi}$. This completes the proof.

\section{REFERENCES}

[1] J.-L. Colliot-Thélène et J.-J. Sansuc, La $R$-équivalence sur les tores, Ann. Sci. Éc. Norm. Sup., 10 (1977), 175-230.

[2] S. Endo and T. Miyata, Quasi-permutation modules over finite groups, J. Math. Soc. Japan, 25 (1973), 397-421: II, ibid., 26 (1974), 698-713.

[ 3 ] _ - On a classification of the function fields of algebraic tori, Nagoya Math. J., 56 (1975), 85-104; Corrigendum, ibid., 79 (1980), 187-190. 
[4] - On integral representations of finite groups, Sugaku, 27 (1975), 232-240 (Japanese).

[5] V. E. Voskresenskiř, Stable equivalence of algebraic tori, Izv. Akad. Nauk SSSR, 38 (1974), 3-10.

Department of Mathematics

Tokyo Metropolitan University

Fukazawa, Setagaya-ku, Tokyo, Japan

Department of Mathematics

Osaka City University

Sugimoto, Sumiyoshi-ku

Osaka-shi, Japan 